\title{
The generalized PRE: Within-S PRF and CRF training in different runways, at different times of day, by different experimenters'
}

\author{
MICHAEL E. RASHOTTE AND ABRAM AMSEL, \\ DEPARTMENT OF PSYCHOLOGY, UNIVERSITY OF \\ TORONTO, Toronto 5, Canada
}

An attempt was made to produce a within-S PRE by running the PRF and CRF trials to patterns of stimulation which differed very greatly. Under these within-S conditions there was still no evidence for a PRE; however, between-S groups run under these conditions showed a clear PRE.

Several recent within-S experiments have shown that rats given PRF training in relation to, say, a black runway and CRF to a white are equally resistant to extinction in both, whether extinction is in both runways or in only one or the other (e.g., Amsel, Rashotte, \& MacKinnon, 1966; Brown \& Logan, 1965; Galbraith, Rashotte, \& Amsel, in press; Spear \& Spitzner, 1967). This within-S finding has been called the generalized partial reinforcement effect (GPRE), and one explanation has been in terms of mediated generalization in extinction (e.g., Amsel, 1967). In the present experiment we tried to break down the GPRE and produce a within-S PRE by increasing ex ternal stimulus control. Ss were trained to run in black and white (otherwise identical) runways as before. However, unlike the earlier experiments in which PRF and CRF trials were run concurrently in each training session, on each day all PRF (or CRF) trials were given in one runway, in one trial-time block, by one $E$, from a particular carrying case, and with a particular condition of background stimulation; and all CRF (or PRF) trials were given $12 \mathrm{~h}$ later by a different $E$, using a different carrying case, and with different background stimulation. Between-S "control" groups were also included.

In many respects our procedures are analogous to those of Asratian (1965). In classical conditioning experiments with dogs, Asratian has demonstrated what he calls "switching": for example, $S$ learns to perform both salivary and defense reflexes to the same CS on the basis of differences in time of day, experimental room, and/or experimenter as discriminative background stimulation.

Subjects. The Ss were 22 male albino rats of the Wistar strain from Woodlyn Farms, Guelph, Ontario, about 120 days of age at the beginning of experimental training.

Apparatus. The apparatus is described in detail by Amsel, Rashotte, \& MacKinnon (1966). Briefly it consisted of two 63-in. long straight runways, one painted flat black and the other flat white, and a common entryboxstartbox unit. A photoelectric system provided five successive 1 - $\mathrm{ft}$ time measures beginning with the opening of the startdoor and ending with the interruption of the last photobeam 3 in. from the food cup.

Procedure. Three weeks prior to the beginning of experimental training, Ss were placed on a $10 \mathrm{~g}$ food deprivation and habituation schedule. All Ss were handled from their home cages for a few minutes each morning by $E_{1}$ and were then fed half their daily ration; $12 \mathrm{~h}$ later $S s$ were handled by $E_{2}$ and were fed the remaining half of their ration. Throughout the experiment, water was freely available in the home cages.

By random assignment four groups were formed. Two groups $(\mathrm{N}=6)$ received within-S training and two $(N=5)$ between-S training. In the within-S condition one group, $\mathrm{B} \pm \mathrm{W} \neq$, was given $50 \%$ reward for runs in the black runway and $100 \%$ reward for runs in the white runway. The other group, $\mathrm{W} \pm \mathrm{B} \neq$, served to provide counterbalancing of the runway brightness and reinforcement factors. In the between-S condition both groups also ran in the black and white runways; the CRF group $(B \neq W \neq)$ found reward on all trials, the PRF group $(\mathrm{B} \pm \mathrm{W} \pm$ ) was rewarded on $50 \%$ of the trials in both the black and white runways.

At each of the two daily experimental sessions all Ss were given four trials in one of the runways and by one of the Es. The experimental sessions were separated by $12 \mathrm{~h}$. For morning sessions, which were preceded by approximately $10 \mathrm{~h}$ of darkness in the colony room, Ss were transported to the experimental room by $E_{1}$ in a 12-place wooden carrying case with masonite doors. The room was quiet and the first of the session's trials was given after a 15-min waiting period. The ITI was $20-25 \mathrm{~min}$. During the session $\mathrm{E}_{1}$ handled Ss with a glove. For the evening session, preceded by $12 \mathrm{~h}$ during which the colony room lights were on and the level of laboratory activity was generally high, $E_{2}$ transported $S s$ to the experimental room in a different 12-place carrying case which had metal doors and was generally newer. In the experimental room a steady loud hum was produced by a fan, and $E_{2}$ handled Ss with a glove different from that used in the morning session. The first of the session's trials was begun after $15 \mathrm{~min}$ of adaptation and the ITI was $20-25 \mathrm{~min}$. At each session Ss were run in two squads of 12 comprised of three Ss from each of the four groups. In each session half of each group was run in the black runway and half in the white.

On rewarded trials, $S$ found one 500-mg Noyes pellet in the food cup. S was removed from the goal after it had consumed the pellet or, on nonrewarded trials, after $30 \mathrm{sec}$ and was returned to the carrying case.

The Ss in each squad were run in a different order on each day but in the same order at both daily sessions. In extinction all procedures were as in acquisition except that food was never present in the goal box.

Results and Discussion. PRF and CRF speeds (1/time) for each of the five runway measures are shown in Fig. 1 for each of the between-S groups and for the combined within-S groups. The within-S data were combined since no significant effects due to subgroups were obtained in analyses of variance conducted on acquisition and extinction data.

Between $-S$. Among the five speed measures, the general pattern of the terminal acquisition data is consistent with Goodrich's (1959) finding that a PRF group receiving $50 \%$ reward runs faster than a CRF group in the early portions of the runway but slower than a CRF group near the goal. Over the last four acquisition days, PRF speeds were reliably higher than CRF speeds in the start measure $(F=7.65, \mathrm{df}=1 / 8, \mathrm{p}<.05)$ and were lower than $\mathrm{CRF}$ speeds in the goal measure $(F=9.05, \mathrm{df}=1 / 8, \mathrm{p}<.05)$. The groups did not differ statistically in the three running measures.

This start-measure finding deserves comment since in two earlier experiments which also included between-S controls (Amsel, Rashotte, \& MacKinnon, 1966, Experiments 3 and 4) no evidence of a PRF crossover was found in the between-S comparisons. In the light of the present finding, it does not seem likely that failure to observe the crossover in the previous experiments was due either to lack of prefeeding experiences in the runway or to exposure of Ss to two runway colors as was suggested by Amsel et al.

In extinction the PRE was evident in all measures and was statistically reliable. These data simply confirm that under the present conditions a PRE will occur after between-S training.

Within-S. Terminal acquisition speeds in the PRF alley were lower than in the CRF in the Run 2, Run 3 and Goal measures and these differences were confirmed in analyses of variance over the last four acquisition days, $F$ 's $=5.19,7.79$ and $13.08, \mathrm{df}=1 / 10$, p's $<.05$, $<.05$, and $<.01$, respectively, for the three measures. There were no reliable differences due to reinforcement in the Start and Run 1 measures. The within-S acquisition data failed to show the PRF crossover which is often, but not always, found under more conventional within-S training conditions (e.g., Amsel, Rashotte, \& MacKinnon, 1966). The speed data in extinction show that performance in the PRF and CRF runways differed very little. Analyses of variance yielded a significant reinforcement effect in the Run 2, Run 3 and Goal measures (F's $=5.35,7.17$, 12.62 , df's $=1 / 10$, p's $<.05,<.05$ and $<.01$, respectively). These effects reflect maintenance of the terminal accuisition differences between the PRF and CRF speeds and do not indicate a difference in rate of extinction (i.e., a PRE). These extinction data are similar to those obtained in earlier within-S experiments in which differences in brightness of the runways, alone, signalled PRF and CRF reward schedules (Amsel, Rashotte, \& MacKinnon, 1966).

The failure of the present within-S extinction data to show more rapid extinction in the CRF than in the PRF runway constitutes yet another demonstration of what Brown \& Logan (1965) have called the generalized PRE. However, it is clear that 


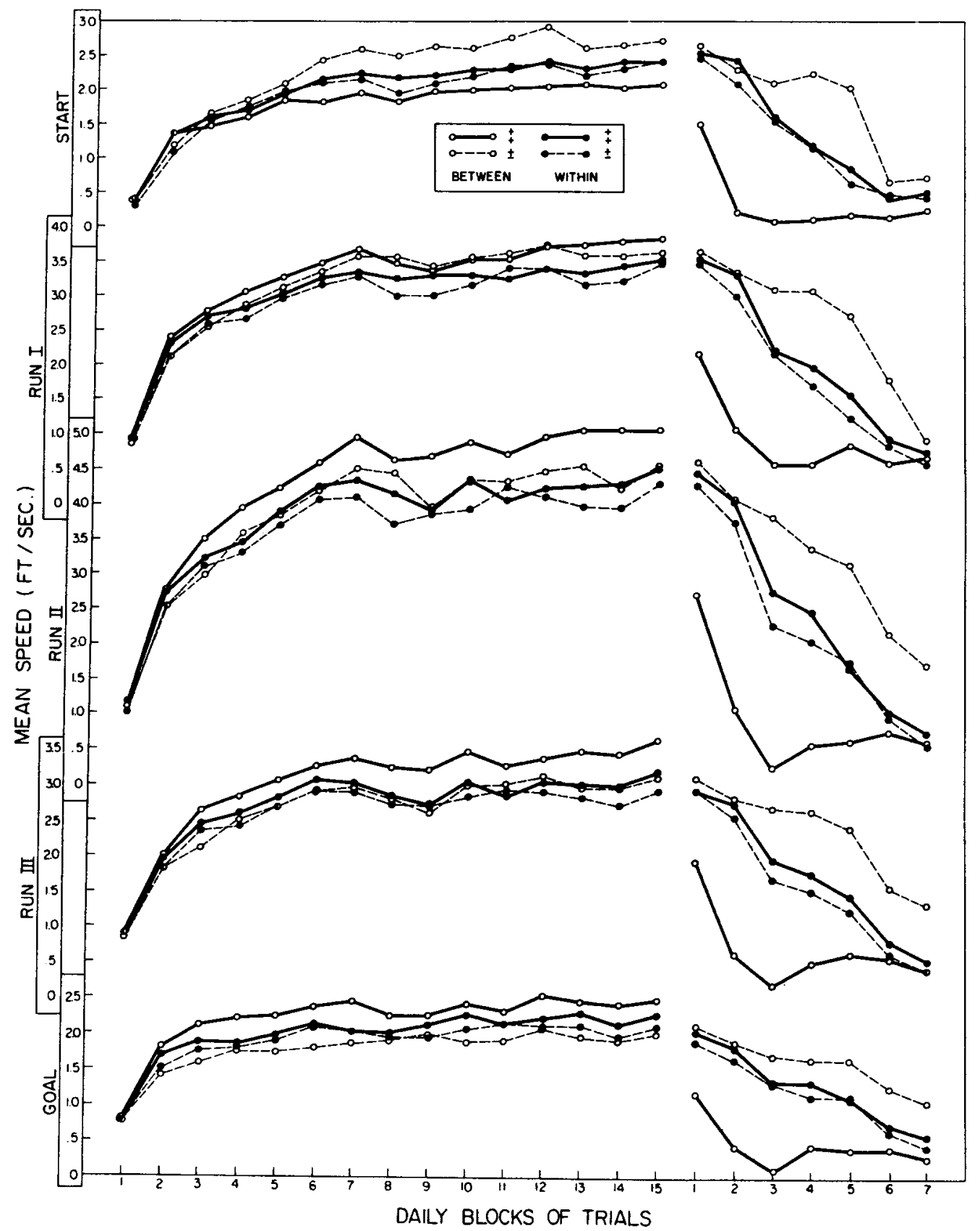

Fig. 1. Within-S and between-S acquisition and extinction across five 1-ft alley segments.

from the third point in extinction onward the two within-S curves fall more sharply than the between-S PRF curve. This suggests that the GPRE designation for the within-S effect may not be entirely appropriate, and that the specific extinction pattern shown in the within-S condition may reflect generalization not only of effects from the PRF to the CRF stimulus but also from the CRF to the PRF (Rashotte, Ross, \& Amsel, in press).

\section{REFERENCES}

AMSEL, A. Partial reinforcement effects on vigor and persistence: advances in frustration theory derived from a variety of within-subjects experiments. In K. W. Spence and J. T. Spence (Eds.), The Psychology of learning and motivation, Vol J. (Advances in research and theory). New York: Academic Press, 1967.

AMSEL, A., RASHOTTE, M. L., \& MacKINNON, J. R. Partial reinforcement effects within subject and hetween subjects. Psychol. Monogr., 1966, 80, (20, Whole No. 628).

ASRATIAN, L. A. Compensatory adaptations, reffex activity, and the brain. London: Pergamon Press, 1965.
BROWN, R. T., \& LOGAN, F. A. Generalized partial reinforcement effect. $J$. comp. physiol Psychol, 1965, 60, 64-69.

GALBRAITH, KAREN, RASHOTTE, M. E., \& AMSEL, A. Within-subjects partial reinforcement effects varying percent reward to the partial stimulus between groups. J. exp. Psychol, in press.

GOODRICH, K. P. Performance in different segments of an instrumental response chain as a function of reinforcement schedule. J. exp. Psychol., $1959,57,57-63$.

PAVLIK, W. B., CARLTON, P. L., \& HUGHES, R. A. Partial reinforcement effects in a runway: between- and within-Ss. Psychon. Sci, 1965, 3, 203-204.

RASHOTTE, M. E., ROSS, M., \& AMSEL, A. Generalization of the partial reinforcement effect. Psychon. Sci, in press.

SPEAR, N. E., \& SPITZNER, J. H. PRE in a T-maze brightness discrimination within and between Ss. J. exp. Psychol, 1967, 73, 321-322.

\section{NOTE}

1. Supported by Research Grants GB-3772 from the National Science Foundation and APB-72 from the National Rescarch Council of Canada. 